\title{
ESTIMACIÓN DE LA \\ HUELLA ECOLÓGICA \\ EN ESTUDIANTES \\ DEL PROGRAMA DE \\ ADMINISTRACIÓN \\ DE EMPRESAS DE \\ LA UNIVERSIDAD \\ DE LA AMAZONIA
}

\section{RESUMEN}

Estimar la huella ecológica de la comunidad académica referente al servicio educativo e identificar sus factores determinantes es sustancial, ya que, además de estimar las emisiones de carbono que contribuyen al calentamiento global, permite identificar las acciones requeridas para disminuir el impacto y sensibilizar al estudiante sobre la contaminación ambiental y el compromiso que debe tener para reducirla. Los resultados muestran que la huella ecológica de un estudiante de Administración de Empresas equivale, en promedio, a 0,89 toneladas de $\mathrm{CO}_{2}$ por semestre, lo cual se encuentra relacionado con el ingreso monetario del estudiante. Este valor es inferior a los hallados en instituciones de educación superior de países desarrollados. Este trabajo se enmarca en los mecanismos para evidenciar el cumplimiento de los principios de desarrollo sostenible en la Universidad de la Amazonia.

Palabras clave: Huella ecológica, Administración de Empresas, Modelo econométrico, Dióxido de carbono. 


\section{YELLY YAMPARLI PARDO ROZO* \\ MILTON CÉSAR ANDRADE ADAIME** \\ SERGIO ANDRÉS CETINA MARTIINEZ***}

\section{ESTIMATION OF THE ECOLOGICAL FOOTPRINT IN STUDENTS OF BUSINESS ADMINISTRATION AT UNIVERSIDAD DE LA AMAZONÍA}

\section{ABSTRACT}

Estimate the ecological footprint of the academic community in the educational service and identifying its determining factors is important because, in addition to estimating the carbon emissions that contribute to global warming, it allows identifying the actions required to reduce the impact and sensitizing the student about the environmental pollution, and the commitment he must have to reduce it. The results show that the ecological footprint of a student of Business Administration is equivalent, on average, to 0.89 tons of $\mathrm{CO}_{2}$ per semester, which is related to the student's monetary income. This value is lower than those found in Higher Education Institutions in developed countries. This work is part of the mechanisms to demonstrate compliance with the principles of sustainable development at the University of the Amazonia.

Keywords: Ecological Footprint, Business Administration, Econometric Model, Carbon Dioxide.

\section{INTRODUCCIÓN}

El calentamiento global es el aumento de la temperatura promedio del planeta a causa de la emisión de gases de efecto invernadero de origen antrópico dadas las actividades de producción y consumo. La consecuencia directa es la redistribución climática en el globo, lo que afecta el equilibrio de los ecosistemas que no pueden recuperarse ante las nuevas condiciones ambientales, sumado esto a las constantes presiones de la extracción y disposición final de desechos. La huella ecológica mide la cantidad de dióxido de carbono $\left(\mathrm{CO}_{2}\right)$ emitido por una actividad en cualquier tipo de organización, para este caso, un servicio educativo, y es una herramienta importante para establecer el impacto de las actividades humanas en los ecosistemas, de forma que permite identificar las medidas y correctivos para mitigar el daño.

Para regular las actividades académicas que impactan el ambiente, la Universidad de la Amazonia cuenta con una política ambiental que responde al cumplimiento de los principios de desarrollo sostenible de los cuales trata la Ley 99 de 1993 en Colombia.

En el marco de la política ambiental de la Uniamazonia, este artículo presenta un

\footnotetext{
* Estudiante de Doctorado, Profesora Universidad de la Amazonia. Colombia. Correo-e: ypardo@udla.edu.co

* Magíster, Profesor Universidad de la Amazonia. Colombia. Correo-e: mandrade@udla.edu.co

*** Administrador de Empresas. Universidad de la Amazonia. Colombia. Correoe: sergiosacm91@hotmail.com

Recibido: 30 de enero de 2017, aceptado: 19 de mayo de 2017.

Para citar el artículo: Pardo, Y. Y.; Andrade, M. C. y Cetina, S. A. (2017). "Estimación de la huella ecológica en estudiantes del programa Administración de Empresas de la Universidad de la Amazonia", en: Sotavento MBA, n. ${ }^{\circ} 29$, pp. 18-28 DOI: http://dx.doi.org/10.18601/01233734.n29.03
} 
ejercicio exploratorio para estimar la huella ecológica de los estudiantes del Programa de Administración de Empresas (PAE), dentro del escenario educativo, mediante la demanda de carbono, energía, agua, papel y su equivalente en árboles, para observar cómo un servicio educativo impacta el medioambiente.

De igual forma, el estudio contempla un modelo empírico fundamentado en el análisis de regresión, que busca relacionar los factores que determinan el aumento o disminución de la huella ecológica con los factores académicos, institucionales y socioeconómicos de los estudiantes del PAE.

\section{FUNDAMENTO TEÓRICO}

La huella ecológica es una herramienta para establecer el impacto de las actividades humanas en los ecosistemas, que ayuda a tomar medidas y correctivos. También es un indicador de la capacidad de integración y operatividad, basado en la eficiencia del uso de energía en diferentes sectores de la economía. La huella ecológica aplicada a la empresa permite reducir las emisiones de $\mathrm{CO}_{2}$ y combate el cambio climático (Ibarra-Cisneros y Monroy, 2014; Doménech, 2010).

La World Wide Fund for Nature - WWF (2012) establece que aunque no es una medida de la población de especies, la huella ecológica provee un indicador de la presión ejercida sobre los ecosistemas y la biodiversidad, mediante la medición del nivel de demanda ecológica de los humanos sobre la biosfera.

Por su parte, Global Footprint Network (2014) afirma que la huella ecológica mundial muestra que la humanidad está usando recursos y produciendo emisiones de $\mathrm{CO}_{2}$ a un ritmo $44 \%$ mayor que el que la naturaleza necesita para reabsorber y regenerarse. Esto se conoce como exceso ecológico y aparece por el agotamiento del capital natural del cual todas las especies dependen para su supervivencia, con profundas implicaciones para los ecosistemas y las especies que ellos soportan, así como para el bienestar y la estabilidad económica de la sociedad.

La huella ecológica de la humanidad ha crecido en un $80 \%$ durante las últimas cuatro décadas. Mientras más grande sea la brecha entre la demanda humana y la capacidad regenerativa o resiliencia de la Tierra, mayor será la presión que habrá en los recursos que otras especies necesitan para sobrevivir y mayor será el número de biodiversidad amenazada y en vía de extinción.

Según Berners-Lee (2010), el calentamiento global es causado por la liberación de ciertos gases a la atmósfera, siendo el $\mathrm{CO}_{2}$ el más dominante cuando se queman combustibles fósiles en hogares, fábricas o plantas de energía, y por la emisión de otros gases de efecto invernadero también importantes como el metano $\left(\mathrm{CH}_{4}\right)$, producido principalmente por la agricultura y los vertederos de basura, el cual es 25 veces por kilogramo más potente que el $\mathrm{CO}_{2} ;$ de igual forma, el óxido nitroso $\left(\mathrm{N}_{2} \mathrm{O}\right)$ es 300 veces más potente que el $\mathrm{CO}_{2}$ y es emitido principalmente por las industrias y la agricultura, y los gases refrigerantes, los cuales son miles de veces más potentes que el $\mathrm{CO}_{2}$ (Peters et al., 2013).

El estándar global para un país en condiciones de desarrollo humano sostenible es de 1,8 ha/cápita como indicador de huella ecológica. Según datos de las Cuentas Nacionales de Huella Ecológica y el Departamento Nacional de Planeación (2011), Colombia es el $26^{\circ}$ país más grande del mundo por área, y el $14^{\circ}$ más grande en capacidad biológica. La huella ecológica en Colombia, 1,9 hectáreas globales per cápita (gha/cápita), es comparable a la de otros países de la Comunidad Andina de Naciones, tales como Bolivia $(2,4)$, Ecuador $(1,9)$ y Perú $(1,8)$. Esto indica la prioridad que debe darse al establecimiento y aplicación de 
medidas encaminadas a prevenir el aumento de dicha huella y, de esta manera, no poner en riesgo la biocapacidad de proveer los servicios ecosistémicos que requieren la sociedad y la economía del país.

La huella de carbono podría ser muy compleja si se expresara en cada uno de los diferentes gases; por ello, lo convencional es expresarla en términos de dióxido de carbono equivalente en $\mathrm{kg}$.

La siguiente investigación busca determinar el equivalente en $\mathrm{CO}_{2}$ por kilogramo generado por las actividades educativas realizadas por los estudiantes del PAE relacionadas con su formación profesional. Además, se busca determinar los factores socioeconómicos y académicos que la determinan.

\section{METODOLOGÍA}

El estudio es del tipo exploratorio y utiliza un diseño descriptivo simple, cuya finalidad es definir el perfil promedio de los estudiantes en las actividades educativas en el consumo de papel, energía, agua y generación de desechos y basuras, para luego asociar su equivalente en $\mathrm{CO}_{2}$ de conformidad con estudios e investigaciones científicas. En su fase descriptiva, el estudio involucra variables cuantitativas y se enfoca más en la amplitud que en la profundidad, no se basa solo en la información de fuentes primarias, sino también en fuentes secundarias.

Para las variables de tipo cuantitativo, se realizó un análisis de parámetros (promedio, mediana, moda, desviaciones estándar, máximos y mínimos); para las variables cualitativas se emplearon tablas de frecuencia. Luego, se hizo una propuesta para la estimación de la huella ecológica a través de un nivel de contaminación dadas las actividades académicas del PAE.

\section{INFORMACIÓN DE FUENTE PRIMARIA}

Para capturar la información, se aplicó la técnica de encuesta. Las encuestas contienen tres bloques de preguntas: características socioeconómicas, características académicas del encuestado y actividades de impacto para el cálculo de la huella ecológica. El cuestionario empleó preguntas de selección múltiple con respuesta única y una pregunta abierta. La encuesta se fundamentó en los aspectos ambientales de que tratan los lineamientos de la Norma Técnica Colombiana ISO 14000.

\section{INFORMACIÓN DE FUENTE SECUNDARIA}

Como principales insumos, se tienen el Plan de Gestión Ambiental de la Universidad (Acuerdo 023 de 2008), los informes anuales de evaluación y la base de datos de la Oficina de Planeación de la Universidad de la Amazonia de 2009 a 2013 para la fase diagnóstica. Otro documento importante es el estudio de García y Vargas (2014), denominado "Factores determinantes del conocimiento, interés y participación en temas de responsabilidad social ambiental en el Programa Administración de Empresas", para contrastar qué determina el interés del estudiante por los temas ambientales.

En el caso del cálculo de la huella ecológica, se utilizaron herramientas online, que incluyen fuentes especializadas en brindar información ambiental como páginas web y libros digitales citados en las referencias bibliográficas.

\section{POBLACIÓN Y MUESTRA}

La unidad de estudio comprende a los estudiantes del programa Administración de Empresas de la Universidad de la Amazonia, sede Florencia, desde el ciclo de profesionalización hasta el ciclo de profundización ( $\mathrm{V}$ a $\mathrm{x}$ semestres). 
A 2014, se contó con una población de 495 matriculados.

El tipo de muestreo es proporcional y se considera que mínimo un 30\% de la población en cada grupo o semestre proporciona información relevante (Jany, 1994), para una muestra de 179 estudiantes.

Para estimar la huella ecológica, primero se hizo un reconocimiento del perfil de actividades educativas de los estudiantes para observar los consumos de agua, papel, energía, generación de desechos plásticos, orgánicos e inorgánicos, y demanda de productos. Luego, se aproximó la demanda de $\mathrm{CO}_{2}$, agua, papel y árboles, fundamentado en equivalencias de diferentes estudios científicos.

\section{MODELO EMPÍRICO}

La variable dependiente es la huella de carbono, la cual se mide en kilogramos de gas carbónico por semestre emitido por el estudiante en las actividades educativas.

Huella de carbono $=\beta_{0}+\beta_{1}{ }^{*}$ género + $\beta_{2}{ }^{*}$ ingresos $+\ldots, \beta_{8}$ * interés ambiental $+\mu$ (Ecuación 1. Modelo empírico)

\section{LAS VARIABLES INDEPENDIENTES SON:}

Género es una variable dummy que toma el valor de 1 si el estudiante es hombre y el valor de 0 si el estudiante es mujer. Ingresos y gastos mensuales son variables continuas medidas en pesos de 2014; rendimiento académico es el promedio acumulado del estudiante (entre 0 y 5); el semestre (5 a 10); la edad en años; estado civil (soltero o casado), y el número de hijos. Se emplea un modelo lineal y el método de máxima verosimilitud para la estimación de los parámetros del modelo. La letra $\mu$ representa el término de error o el componente probabilístico.

\section{RESULTADOS}

\section{CÁLCULO DE LA HUELLA ECOLÓGICA PARA LOS ESTUDIANTES DEL PAE}

Se halló la huella ecológica a partir del consumo de papel, energía, transporte, alimentos, bebidas, vestuario, agua, elementos como CD, lapiceros, agendas, bolsos y la emisión realizada por los fumadores, residuos sólidos generados en actividades relativas al servicio educativo en un periodo semestral.

\subsection{PAPEL}

Un estudiante del PAE invierte en promedio $\$ 6.008$ pesos $\mathrm{m} / \mathrm{cte}$ en fotocopias por semana. Si se tiene en cuenta que el valor promedio actual de una fotocopia es de $\$ 60$, entonces, un estudiante del PAE hace uso de 1.602 fotocopias por semestre.

Suponiendo que las 1.602 fotocopias sean utilizadas por las dos caras de la hoja, se puede afirmar que un estudiante PAE hace uso de 801 hojas por concepto de fotocopias por semestre. Por lo tanto, la totalidad de hojas por semestre que generan todos los estudiantes PAE (805 estudiantes) en fotocopias es 644.859, lo que equivale a cerca de 1.290 resmas de papel.

Según Clean Up Australia (2009), se usan aproximadamente 90.000 litros de agua para producir 1 tonelada $(1.000 \mathrm{~kg})$ de papel. En el caso de los estudiantes del PAE, las 644.859 hojas equivalen a $4.030,37 \mathrm{~kg}$, teniendo en cuenta información de Carvajal Tecnología y Servicios (2014). Esto indica que para producir la totalidad de las hojas que se consumen semestralmente por concepto de fotocopias en la Universidad de la Amazonia se necesitarían 362.733 litros de agua. 
Adicionalmente, Visy Recycling (2014) afirma que una tonelada de papel consume aproximadamente 20 árboles adultos. Es decir que los 4.030,37 kilogramos de hojas de papel de fotocopias semestrales del PAE equivalen a talar 81 árboles. Para compensar este daño, se tendrían que sembrar 162 árboles anualmente por parte de los estudiantes del programa. Al producir $200 \mathrm{~kg}$ de papel, se emiten $160 \mathrm{Kg}$ de $\mathrm{CO}_{2}$, lo que quiere decir que para producir los $4.030,37 \mathrm{~kg}$ de papel que se consumen en el PAE para fotocopias de los estudiantes, se emiten aproximadamente $3.224 \mathrm{~kg}$ de $\mathrm{CO}_{2}$. De esto se infiere que para fabricar el papel que usa en fotocopias cada estudiante del PAE, se emitieron cerca de $4 \mathrm{~kg}$ de $\mathrm{CO}_{2}$.

Por otra parte, Aspapel (2010) informa que un kilogramo de papel almacena 1,3 kilogramos de $\mathrm{CO}_{2}$. Esto significa que cuando se descompone todo el papel de fotocopias de un semestre de los estudiantes del PAE, se están emitiendo $5.239 \mathrm{~kg}$ de $\mathrm{CO}_{2}$.

El mismo ejercicio indica que para producir las agendas, libretas o cuadernos que compran los estudiantes del PAE semestralmente se requieren alrededor de 45.281 litros de agua y 10 árboles, se emiten $402,50 \mathrm{~kg}$ de $\mathrm{CO}_{2}$ y se encuentran almacenados cerca de 654,06 $\mathrm{kg}$ de $\mathrm{CO}_{2}$ que serán liberados a la atmósfera cuando se descompongan.

Por lo anterior, el consumo de papel de los estudiantes del PAE contribuye con la emisión de $3.626,80 \mathrm{~kg}$ de $\mathrm{CO}_{2}$, con el uso de 408.014,44 litros de agua y con la tala de 90,67 árboles por semestre. Además, en el papel que se utiliza, se encuentran almacenados $5.894 \mathrm{~kg}$ de $\mathrm{CO}_{2}$ que serán emitidos posteriormente.

De lo anterior se deduce que, cada semestre, un estudiante contribuye con la emisión de 4,5 kg. de $\mathrm{CO}_{2}$, el uso de 506,85 litros de agua, la tala de 0,11 árboles y con 7,32 kg de $\mathrm{CO}_{2}$ almacenado.

\subsection{ENERGÍA}

La suma de las emisiones correspondientes a los aspectos evaluados que tienen que ver con el consumo de energía de los estudiantes PAE, como las emisiones provenientes del uso de energía trabajando en el portátil y computador de escritorio, por uso de la bombilla para estudiar, por uso de teléfono móvil, por cargar el celular, uso de video beam y ventilador o aire acondicionado, se resume en los siguientes puntos:

- Producir los computadores portátiles: $31.918 \mathrm{~kg}$ de $\mathrm{CO}_{2}$ al ambiente.

- Uso de la energía para estudiar en el portátil: los 523 estudiantes que tienen portátil están emitiendo $8.633 \mathrm{~kg}$ por semestre.

- $17.688 \mathrm{~kg}$ de $\mathrm{CO}_{2}$ por uso del computador de escritorio en casa.

- Uso de la bombilla en la noche: 5,04 kg de $\mathrm{CO}_{2}$ y $4.057,2 \mathrm{~kg}$ por el total de los estudiantes.

- $1.893 \mathrm{~kg}$ de $\mathrm{CO}_{2}$ por uso de la bombilla en el día.

- Para la fabricación de los celulares de los estudiantes PAE, se emitieron alrededor de 12.496 kilogramos de $\mathrm{CO}_{2}$.

- Uso del celular: $268.333 \mathrm{~kg}$ de $\mathrm{CO}_{2}$.

- $624 \mathrm{Kg}$ por cargar los celulares cada semestre.

- $224 \mathrm{Kg}$ de $\mathrm{CO}_{2}$ por semestre por uso de video beam.

- $4.491 \mathrm{Kg}$ por uso del ventilador.

Los valores fueron referenciados bajo los estudios de Climate Communication (2012). El total de emisiones de $\mathrm{CO}_{2}$ de los estudiantes del PAE por concepto de energía ascienden a $350.357 \mathrm{~kg}$ por cada semestre. 


\subsection{TRANSPORTE}

Según Bloomberg (2014), junto con el grupo Associates for International Research Inc. - AIRINC, un galón de gasolina equivale a 3,785411784 litros (redondeado a 3,7854 litros).

Teniendo en cuenta que el $71 \%$ de los estudiantes del PAE se desplazan en motocicleta a la universidad ( $53 \%$ en moto propia y el $18 \%$ en mototaxi), se calcula que este porcentaje equivale a 572 estudiantes del total de 805. Asimismo, según Low Carbon Vehicle Partnership (2010), una motocicleta emite en promedio $110 \mathrm{~g}$ de $\mathrm{CO}_{2}$ por kilómetro recorrido.

Según la encuesta, un estudiante del PAE va en promedio 2 veces a la universidad y se demora 14 minutos en un trayecto, lo que muestra que los 4 trayectos equivalen a 56 minutos de desplazamiento diario por estudiante. Durante estos minutos se emiten cerca de $2.200 \mathrm{~g}$ de $\mathrm{CO}_{2}$. De lo anterior se infiere que cada estudiante emite $264 \mathrm{~kg}$ de $\mathrm{CO}_{2}$ y el total de estudiantes del PAE que utilizan motocicletas como medio de transporte son responsables de la emisión de $151.008 \mathrm{~kg}$ de $\mathrm{CO}_{2}$ semestrales.

Por otra parte, el $7 \%$ se desplaza en carro ( $5 \%$ en auto propio y el $2 \%$, en taxi). Este porcentaje equivale a 56 estudiantes. De acuerdo con Metro Madrid (2013) y con Low Carbon Vehicle Partnership, un carro emite aproximadamente 189 gramos de $\mathrm{CO}_{2} / \mathrm{km}$. Es decir que cada estudiante que va en promedio dos veces a la universidad (4 viajes), estaría emitiendo cerca de $453 \mathrm{~kg}$ de $\mathrm{CO}_{2}$ por cada semestre y el total de estudiantes que usan carro como medio de transporte estarían generando $25.402 \mathrm{~kg}$ de dióxido de carbono.

De igual forma, el $4 \%$ de los estudiantes PAE utiliza el bus como medio de transporte. Según información de Werner (2008), por cada pasajero se emiten cerca de $80 \mathrm{~g}$ de $\mathrm{CO}_{2}$ por kilómetro recorrido del bus. Es decir, que cada uno de los 32 estudiantes que utilizan este medio estarían generando una huella de aproximadamente $192 \mathrm{~kg}$ de $\mathrm{CO}_{2}$ por cada semestre de su carrera y el total de estudiantes emitiría cerca de $6.144 \mathrm{~kg}$ de $\mathrm{CO}_{2}$.

El total de emisiones por concepto de transporte de los estudiantes del PAE incluye los $151.008 \mathrm{~kg}$ de $\mathrm{CO}_{2}$ semestrales de los que utilizan motocicletas, 25.402 de quienes usan el carro y los 6.144 de $\mathrm{CO}_{2}$ de quienes toman el autobús. El total asciende a $182.554 \mathrm{~kg}$ de $\mathrm{CO}_{2}$ por cada semestre.

\subsection{OTROS IMPACTOS}

Con el humo del cigarrillo, se emiten 16,2 gramos de $\mathrm{CO}_{2}$ a la atmósfera cada semestre, y el total de estudiantes del PAE estaría emitiendo $13,041 \mathrm{~kg}$. Las prendas de vestir estarían emitiendo cerca de 95,06 kg durante todo su ciclo de vida, lo que indica que el $\mathrm{CO}_{2}$ emitido por todos los estudiantes del PAE por concepto de compra de ropa es de cerca de $76.522 \mathrm{~kg}$.

Teniendo en cuenta que, según los resultados de la encuesta realizada, un estudiante del PAE consume aproximadamente 1,4 veces en el día en las cafeterías de la universidad, se puede afirmar que los estudiantes consumen al menos una de las tres comidas diarias en la institución. Según Scarborough et al. (2014), una persona que tiene una dieta acompañada de muchos productos y derivados cárnicos tiene una huella de carbono diaria de aproximadamente $7,19 \mathrm{~kg}$, mientras que una persona vegetariana contribuye a las emisiones de aproximadamente $3,18 \mathrm{~kg}$ y un vegano solo emitiría diariamente $2,89 \mathrm{~kg}$ de $\mathrm{CO}_{2}$ por concepto de sus alimentos diarios.

Teniendo en cuenta que los productos que se encuentran en las cafeterías de la Universidad tienen alto contenido de carne, como las empanadas, chorizos, papas rellenas, suizos, hamburguesas, perros calientes, etc., se asume que los estudiantes del PAE se encuentran en la 
categoría de consumidores de carne, los cuales emiten $7,19 \mathrm{~kg}$ de carne diaria (incluidas las bebidas). Es decir que la comida que ingieren los estudiantes en las cafeterías de la universidad equivaldría a $1 / 3$ del total, lo cual corresponde a aproximadamente $2,4 \mathrm{~kg}$ de $\mathrm{CO}_{2}$ diarios. En el semestre, suman 287,6 kilogramos de dióxido de carbono por cada estudiante y por el total de alumnos son $231.518 \mathrm{~kg}$ de $\mathrm{CO}_{2}$.

\subsection{AGUA}

La duración de una ducha en promedio es de 14 minutos. El estudiante del PAE usa cerca de 75.600 litros de agua para ducharse cada semestre. Los baños antiguos usan entre 3,5 y 5 litros de agua por descarga y los nuevos llegan a ser tan eficientes que usan entre 1,28 y 1,6 litros. El total de agua utilizada por todos los estudiantes cada semestre por uso de los baños de la universidad está por encima de 322.000 litros.

\subsection{RESIDUOS SÓLIDOS}

Para Kulpinski (2008), la huella que genera una persona no termina después de que se compran y se consumen las cosas; el impacto final ocurre cuando se descartan los objetos. En América Latina, el promedio de basura por día es de 1 kilogramo, lo cual equivale a más de 360 en el año por una sola persona.

Para estimar cuánta basura genera en total cada estudiante del PAE, se tuvo en cuenta el análisis de las encuestas aplicadas, donde se pudo observar que 1 estudiante produce alrededor de 0,508 kilogramos de basura al día, 0 sea que cada estudiante genera 91,44 kilogramos de basura por semestre. Esto indica que la totalidad de los estudiantes (805), estarían generando cerca de 73.609,2 kilogramos de basura por cada semestre.

\section{EL PLÁSTICO}

Según el análisis de los resultados de las encuestas aplicadas a los estudiantes, se observa que la mayoría consume 1 botella de agua de $600 \mathrm{ml}$; esto indica que son 805 botellas en total. Por otra parte, de acuerdo con Cagliani (2011), se puede decir que 1 botella plástica (PET) de $600 \mathrm{ml}$ emite 160 gramos de $\mathrm{CO}_{2}$ i haciendo la conversión, tenemos que 1 kilogramo de plástico ( $P E T$ ) emite 0,16 kilogramos de $\mathrm{CO}_{2}$. Teniendo en cuenta que la población del programa es de 805 estudiantes, entonces, las emisiones que se generan por el consumo de agua en botellas son 128,8 kilogramos de $\mathrm{CO}_{2}$. Por lo tanto, semestralmente, se estarían generando cerca de $23.184 \mathrm{~kg}$ de dióxido de carbono.

Para elaborar una botella (PET) de 600 $\mathrm{ml}$ se necesitan 17,5 kilogramos de agua, lo cual indica que para producir las 805 botellas que consumen los estudiantes del PAE se utilizaron 14.087, 5 litros de agua.

Según estudios realizados por el científico Bill Bryson y el World Watch Institute (2004), producir un litro de agua embotellada genera hasta 600 veces más $\mathrm{CO}_{2}$ que un litro de agua del grifo; esto indica que mientras menos agua en botella se consuma, menos serán las emisiones de $\mathrm{CO}_{2}$ a la atmósfera.

Los estudiantes acumulan alrededor de 60,37 kilogramos de desechos con estos discos compactos (CD). En promedio, demandan al semestre 3 unidades. Igualmente, cada estudiante del PAE utiliza en promedio 5 lapiceros por semestre, esto es, 4.025 lapiceros para el total de estudiantes. Cada lapicero pesa alrededor de 8 gramos, que equivalen a 0,008 kilogramos, entonces, el peso total de los lapiceros utilizados es de 32,2 kilogramos.

Según la General Electric Company (2014), se calcula que una persona emite 230 kilogramos de $\mathrm{CO}_{2}$ de desechos de basura por año. Entonces, por semestre, un estudiante 
emite alrededor de 115 kilogramos de $\mathrm{CO}_{2}$. El total de estudiantes del PAE sumarían 92.575 kilogramos de $\mathrm{CO}_{2}$ por semestre.

\subsection{DETERMINANTES DE LA HUELLA ECOLÓGICA}

El factor determinante de la huella ecológica, desde el punto de vista estadístico, fue el ingreso del estudiante.

Tabla 1. Modelo empírico obtenido por mínimos cuadrados ordinarios, función lineal

\begin{tabular}{|l|c|c|}
\hline \multicolumn{1}{|c|}{ Parámetro } & Coeficiente & Estadístico t \\
\hline Intercepto & 6597,93052 & 2,79 \\
\hline Ingreso & 3,51241709 & 2,89 \\
\hline
\end{tabular}

Fuente: Los autores.

El modelo econométrico arroja como única variable explicativa de la emisión de dióxido de carbono el ingreso, esto es, a mayor ingreso, mayor emisión del gas de efecto invernadero - GEI en las actividades educativas del PAE. Las variables de género, edad, semestre ni estado civil fueron relevantes estadísticamente. Luego, se observa que por cada $\$ 10.000$ pesos que aumente el ingreso en el semestre, el estudiante aumentará sus emisiones de $\mathrm{CO}_{2}$ en 3,51 kilogramos.

\section{CONCLUSIONES Y DISCUSIÓN}

La medición de la huella ecológica indica que un estudiante PAE produce aproximadamente 0,89 toneladas de $\mathrm{CO}_{2}$ y el total de estudiantes contribuye con la emisión de $718.676 \mathrm{~kg}$ de $\mathrm{CO}_{2}$ cada semestre, de acuerdo con sus actividades académicas. Según la medición realizada, la energía es el aspecto que más contribuye con la generación de $\mathrm{CO}_{2}$, seguido del transporte, la producción de desechos, el vestuario, el papel y el humo del cigarrillo, en ese orden. Este valor está por encima de lo reportado por la National University of Lesotho $(0,1$ toneladas de emisiones de $\mathrm{CO}_{2}$ por estudiante) y la Universidad Autónoma de Chile $(0,5)$ e iguala a la City University London $(0,8)$, pero por debajo de la Universidad de Mariland. En cuanto a las variables determinantes, solo el ingreso mensual del estudiante determina las emisiones de $\mathrm{CO}_{2}$.

\section{REFERENCIAS}

Aspapel (2010). Árbol, papel, planeta: un ciclo con el que ganamos todos. España: Información e imagen. Recuperado de: http://www.aspapel. es/sites/default/files/publicaciones/Doc\%2075.pdf

Berners-Lee, M. (2010). What's the carbon footprint of... using a mobile phone. Reino Unido:The Guardian. Recuperado de: http://www.theguardian. com/environment/green-living-blog/2010/jun/09/ carbon-footprint-mobile-phone

Berners-Lee, Mike. (2010). How bad are bananas? The carbon footprint of everything. London, Great Britain: Profile Books Ltd.

Bloomberg (2014). Paint at the Pump: Gasoline by Country. Europa: Bloomberg visual data. Recuperado de: http://www.bloomberg.com/visualdata/gas-prices/

Cagliani, M. (2011). Huella de carbono del plástico. Argentina: Sustentator. Recuperado de: http://www.petreciclados.com.ar/plasticos.php

Carbon Footprint (2014). Reduce, Re-use, Repair and Recycle. Reino Unido. Recuperado de: http://www.carbonfootprint.com/recycling.html

Carvajal Tecnología y Servicios (2014). Justificación de proyecto de papel. México: Carvajaltys. Recuperado de: http://www.levicom.com.mx/flash/ green/st5.swf

Clean Up Australia Ltd. (2009). Paper and Cardboard Fact Sheet. Australia: Cleanup.org. Recuperado de: http://www.cleanup.org.au/PDF/ au/cua_paperandcardboard_fact_sheet_final.pdf

Climate Communication (2012). Energy Use, the Source of Most Carbon Emissions. Estados 
Unidos: Climate notes. Recuperado de: http://www. climatecommunication.org/change/energy-use/

Data visualization (2014). How much $\mathrm{CO}_{2}$ is created by... Estados Unidos: General Electric Company. Recuperado de: http://visualization.geblogs.com/visualization/co2/

Departamento Nacional de Planeación (2011). Plan Nacional de Desarrollo: 2010-2014 ( Tomo I). Prosperidad para todos. Bogotá, Colombia: DNP. Recuperado de: https://colaboracion.dnp. gov.co/CDT/PND/PND2010-2014\%20Tomo\%20 I\%20CD.pdf

Doménech, Q.J. L. (2010). Huella ecológica y desarrollo sostenible. España: AENOR - Asociación Española de Normalización y Certificación. Recuperado de http://www.ebrary.com

Energuide (2012). The answer to all your questions on energy in Brussels. Bélgica: sibelga Recuperado de: http://www.energuide.be/en/questions-answers/how-much-power-does-a-computeruse-and-how-much-co2-does-that-represent/54

Energy Information Administration (2013). International Energy Statistics. Estados Unidos: us Department of Energy. Recuperado de: http://www.eia.gov/cfapps/ipdbproject/IEDIndex3. $\mathrm{cfm}$ ?tid=90\&pid=44\&aid $=8$

EPA (2012). Noise Pollution. Estados Unidos. Recuperado de: http://epa.gov/air/noise.html

EPA (2014). Recycling Basics. Estados Unidos. Recuperado de: http://www2.epa.gov/recycle/ recycling-basics

EPA (2014). Transportation and climate. Estados Unidos. Recuperado de: http://www.epa. gov/otaq/climate/basicinfo.htm

European Union (2010). What your appliances cost you and how much $\mathrm{CO}_{2}$ they emit? Bélgica: European Commission. Recuperado de: http:// ec.europa.eu/clima/sites/campaign/pdf/table_ appliances_en.pdf

García, J. y Vargas, K. (2014). Medición del grado de conocimiento, participación e interés en temas ambientales en estudiantes del Programa Administración de Empresas de la Universidad de la Amazonia. Florencia, Colombia: sin publicar.
General Electric Company (2014).How much $\mathrm{CO} 2$ is created by... Estados Unidos: Data Visualization. Recuperado de: http://visualization. geblogs.com/visualization/co2/\#/kg_waste_person

Global Footprint Network (2014). Living Planet Report: Species and Spaces, People and Places. Suiza: Living Planet Index. Recuperado de: http://ba04e385e36eeed47f9c-abbcd57a2a90674a4bcb7fab6c6198d0.r88.cf1.rackcdn. com/Living_Planet_Report_2014.pdf

Ibarra-Cisneros, J. M.y Monroy-Ata A. (2014). Cuestionario para calcular huella ecológica de estudiantes universitarios mexicanos y su aplicación en el campus Zaragoza de la Universidad Nacional. Recuperado el 29 de septiembre de 2015 de: http:// www.elsevier.es

Kulpinski, D. (2008). Human Footprint: Where Does All the Stuff Go? Estados Unidos: National Geographic. Recuperado de: http://channel. nationalgeographic.com/channel/human-footprint/ trash-talk.html

Low Carbon Vehicle Partnership (2010). Carbon Transport Footprint. Reino Unido: LowCVP. Recuperado de: http://www.lowcvp.org.uk/.../MCI\%20 -\%20Greg\%20Archer

Metro Madrid (2013). Metro passengers generate three times less $\mathrm{CO}_{2}$ than car users. España: Comunidad de Madrid. Recuperado de: https://www. metromadrid.es/en/conocenos/responsabilidad_corporativa/Huella_carbono/index.html

Nickson, C. (2014). Walking and your carbon footprint. Reino Unido: Carbon Counted. Recuperado de: http://www.carboncounted.co.uk/walkingandyourcarbonfootprint.html

Otto Group (2011). Carbon Footprint Measure of Garments. India. Recuperado de: http://www. onlineclothingstudy.com/2011/02/carbon-footprintmeasure-of-garments.html

Peters, Michael et al. (2013). Challenges and opportunidties for improvising eco-efiiciency of tropical forage-based systems to mittigate greenhouse gas emissions. Cali, Colombia: Centro Internacional de Agricultura - CIAT. 
Scarborough, P.; Appleby, P. N.; Mizdrak, A.; Briggs, A. D.; Travis, R. C. y Bradbury, K. E. (2014). Dietary greenhouse gas emissions of meat-eaters, fish-eaters, vegetarians and vegans in the UK. Reino Unido: Climate Change. Recuperado de: http://link. springer.com/article/10.1007\%2Fs10584-0141169-1\#page-1

Steer, W.A. (2007). What would a smokers Carbon footprint look like?. Reino Unido: University of Cambridge. Recuperado de: http://www.thenakedscientists.com/forum/index.php?topic $=11256.0$

Uniamazonia (2008). Plan de Gestión Ambiental: Acuerdo 023 de 2008. Colombia: Universidad de la Amazonia. Recuperado de: http://apps.udla. edu.co/documentos/docs/Oficina\%20Asesora\%20 de \% 20 Planeacion/Sistema \% 20 Integrado \% 20 de\%20Gestion\%20de\%20Calidad/Plan\%20de\%20 gestion\%20ambiental.pdf
Visy Recycling (2014). Transforming Resources for a Better World. Australia: Visy industries: Recuperado de: http://www.visy.com.au/recycling-about/

Werner, M. (2008). Motorcycle and other vehicles $\mathrm{CO}_{2}$ emissions. France: Motorcycle News. Recuperado de: http://news.motorbiker.org/blogs.nsf/ $\mathrm{dx} /$ motorcycles-and-other-vehicles-co2-emissions.htm

World Watch Institute (2004). Indicadores ambientales. España: Junta de Andalucía. Recuperado de: http://www.ecoestrategia.com/articulos/ indicadores/indicadores.html

World Wide Fund for Nature (2012). Living Planet Report 2012: Biodiversity, biocapacity and better choices. Suiza: WWF. Recuperado de: http:// issuu.com/globalfootprintnetwork/docs/living_planet_report_2012?e=2286412/2659274\#222222 